\title{
Sharp bounds for Seiffert mean in terms of root mean square
}

Yu-Ming Chu ${ }^{1 *}$, Shou-Wei Hou ${ }^{2}$ and Zhong-Hua Shen ${ }^{2}$

* Correspondence:

chuyuming2005@yahoo.com.cn

${ }^{1}$ Department of Mathematics and

Computing Science, Hunan City

University, Yiyang 413000, China

Full list of author information is

available at the end of the article

\section{Abstract}

We find the greatest value $\alpha$ and least value $\beta$ in $(1 / 2,1)$ such that the double inequality

$$
S(\alpha a+(1-\alpha) b, \alpha b+(1-\alpha) a)<T(a, b)<S(\beta a+(1-\beta) b, \beta b+(1-\beta) a)
$$

holds for all $a, b>0$ with $a \neq b$. Here, $T(a, b)=(a-b) /[2 \arctan ((a-b) /(a+b))]$ and $S(a$, $b)=\left[\left(a^{2}+b^{2}\right) / 2\right]^{1 / 2}$ are the Seiffert mean and root mean square of $a$ and $b$, respectively.

2010 Mathematics Subject Classification: 26E60.

Keywords: Seiffert mean, root mean square, power mean, inequality

\section{Introduction}

For $a, b>0$ with $a \neq b$ the Seiffert mean $T(a, b)$ and root mean square $S(a, b)$ are defined by

$$
T(a, b)=\frac{a-b}{2 \arctan \left(\frac{a-b}{a+b}\right)}
$$

and

$$
S(a, b)=\sqrt{\frac{a^{2}+b^{2}}{2}},
$$

respectively. Recently, both mean values have been the subject of intensive research. In particular, many remarkable inequalities and properties for $T$ and $S$ can be found in the literature [1-14].

Let $A(a, b)=(a+b) / 2, G(a, b)=\sqrt{a b}$, and $M_{p}(a, b)=\left(\left(a^{p}+b^{\mathrm{p}}\right) / 2\right)^{1 / p}(p \neq 0)$ and $M_{0}(a, b)=\sqrt{a b}$ be the arithmetic, geometric, and $p$ th power means of two positive numbers $a$ and $b$, respectively. Then it is well known that

$$
G(a, b)=M_{0}(a, b)<A(a, b)=M_{1}(a, b)<T(a, b)<S(a, b)=M_{2}(a, b)
$$

for all $a, b>0$ with $a \neq b$.

Seiffert [1] proved that inequalities

$$
A(a, b)<T(a, b)<S(a, b)
$$


hold for all $a, b>0$ with $a \neq b$.

Chu et al. [5] found the greatest value $p_{1}$ and least value $p_{2}$ such that the double inequality $H_{p 1}(a, b)<T(a, b)<H_{p 2}(a, b)$ holds for all $a, b>0$ with $a \neq b$, where $H_{p}(a, b)=$ $\left(\left(a^{p}+(a b)^{p / 2}+b^{p}\right) / 3\right)^{1 / p}(p \neq 0)$ and $H_{0}(a, b)=\sqrt{a b}$ is the $p$ th power-type Heron mean of $a$ and $b$.

In [6], Wang et al. answered the question: What are the best possible parameters $\lambda$ and $\mu$ such that the double inequality $L_{\lambda}(a, b)<T(a, b)<L_{\mu}(a, b)$ holds for all $a, b>0$ with $a \neq b$ ? where $L_{r}(a, b)=\left(a^{r+1}+b^{r+1}\right) /\left(a^{r}+b^{r}\right)$ is the $r$ th Lehmer mean of $a$ and $b$.

Chu et al. [7] proved that inequalities

$$
p T(a, b)+(1-p) G(a, b)<A(a, b)<q T(a, b)+(1-q) G(a, b)
$$

hold for all $a, b>0$ with $a \neq b$ if and only if $p \leq 3 / 5$ and $q \geq \pi / 4$.

Hou and Chu [9] gave the best possible parameters $\alpha$ and $\beta$ such that the double inequality

$$
\alpha S(a, b)+(1-\alpha) \bar{H}(a, b)<T(a, b)<\beta S(a, b)+(1-\beta) \bar{H}(a, b)
$$

holds for all $a, b>0$ with $a \neq b$.

For fixed $a, b>0$ with $a \neq b$, let $x \in[1 / 2,1]$ and

$$
f(x)=S(x a+(1-x) b, x b+(1-x) a) .
$$

Then it is not difficult to verify that $f(x)$ is continuous and strictly increasing in [1/ $2,1]$. Note that $f(1 / 2)=A(a, b)<T(a, b)$ and $f(1)=S(a, b)>T(a, b)$. Therefore, it is natural to ask what are the greatest value $\alpha$ and least value $\beta$ in $(1 / 2,1)$ such that the double inequality

$$
S(\alpha a+(1-\alpha) b, \alpha b+(1-\alpha) a)<T(a, b)<S(\beta a+(1-\beta) b, \beta b+(1-\beta) a)
$$

holds for all $a, b>0$ with $a \neq b$. The main purpose of this article is to answer these questions. Our main result is the following Theorem 1.1.

Theorem 1.1. If $\alpha, \beta \in(1 / 2,1)$, then the double inequality

$$
S(\alpha a+(1-\alpha) b, \alpha b+(1-\alpha) a)<T(a, b)<S(\beta a+(1-\beta) b, \beta b+(1-\beta) a)(1.3)
$$

holds for all $a, b>0$ with $a \neq b$ if and only if $\alpha \leq\left(1+\sqrt{16 / \pi^{2}-1}\right) / 2$ and $\beta \geq(3+\sqrt{6}) / 6$.

\section{Proof of Theorem 1.1}

Proof of Theorem 1.1. Let $\lambda=\left(1+\sqrt{16 / \pi^{2}-1}\right) / 2$ and $\mu=(3+\sqrt{6}) / 6$.

We first proof that inequalities

$$
T(a, b)>S(\lambda a+(1-\lambda) b, \lambda b+(1-\lambda) a)
$$

and

$$
T(a, b)<S(\mu a+(1-\mu) b, \mu b+(1-\mu) a)
$$

hold for all $a, b>0$ with $a \neq b$.

From (1.1) and (1.2), we clearly see that both $T(a, b)$ and $S(a, b)$ are symmetric and homogenous of degree 1 . Without loss of generality we assume that $a>b$. Let $t=a / b$ $>1$ and $p \in(1 / 2,1)$, then from (1.1) and (1.2) one has 


$$
\begin{aligned}
& S(p a+(1-p) b, p b+(1-p) a)-T(a, b) \\
& =b \frac{\sqrt{[p t+(1-p)]^{2}+[(1-p) t+p]^{2}}}{2 \arctan \left(\frac{t-1}{t+1}\right)} \\
& \times\left\{\sqrt{2} \arctan \left(\frac{t-1}{t+1}\right)-\frac{t-1}{\sqrt{[p t+(1-p)]^{2}+[(1-p) t+p]^{2}}}\right\} .
\end{aligned}
$$

Let

$$
f(t)=\sqrt{2} \arctan \left(\frac{t-1}{t+1}\right)-\frac{t-1}{\sqrt{[p t+(1-p)]^{2}+[(1-p) t+p]^{2}}},
$$

then simple computations lead to

$$
\begin{aligned}
& f(1)=0, \\
& \lim _{t \rightarrow+\infty} f(t)=\frac{\sqrt{2 \pi}}{4}-\frac{1}{\sqrt{p^{2}+(1-p)^{2}}}, \\
& f^{\prime}(t)=\frac{f_{1}(t)}{\left\{[p t+(1-p)]^{2}+[(1-p) t+p]^{2}\right\}^{\frac{3}{2}}\left(t^{2}+1\right)},
\end{aligned}
$$

where

$$
f_{1}(t)=\sqrt{2}\left\{[p t+(1-p)]^{2}+[(1-p) t+p]^{2}\right\}^{\frac{3}{2}}-(t+1)\left(t^{2}+1\right) .
$$

Note that

$$
\begin{aligned}
& \left\{\sqrt{2}\left\{[p t+(1-p)]^{2}+[(1-p) t+p]^{2}\right\} \frac{3}{2}\right\}^{2}-\left[(t+1)\left(t^{2}+1\right)\right]^{2} \\
& =(t-1)^{2} g_{1}(t),
\end{aligned}
$$

where

$$
\begin{aligned}
& g_{1}(t)=\left(16 p^{6}-48 p^{5}+72 p^{4}-64 p^{3}+36 p^{2}-12 p+1\right) t^{4}-16 p^{2} \\
& \left(4 p^{2}-4 p+3\right)(p-1)^{2} t^{3}+2\left(48 p^{6}-144 p^{5}+168 p^{4}-96 p^{3}+36 p^{2}-12 p+1\right) \\
& \times t^{2}-16 p^{2}\left(4 p^{2}-4 p+3\right)(p-1)^{2} t+16 p^{6}-48 p^{5}+72 p^{4}-64 p^{3}+36 p^{2} \\
& -12 p+1 \\
& g_{1}(1)=4\left(12 p^{2}-12 p+1\right) .
\end{aligned}
$$

Let $g_{2}(t)=g_{1}^{\prime}(t) / 4, g_{3}(t)=g_{2}^{\prime}(t), g_{4}(t)=g_{3}^{\prime}(t) / 6$. Then simple computations lead to

$$
\begin{aligned}
& g_{2}(t)=\left(16 p^{6}-48 p^{5}+72 p^{4}-64 p^{3}+36 p^{2}-12 p+1\right) t^{3}-12 p^{2} \\
& \left(4 p^{2}-4 p+3\right)(p-1)^{2} t^{2}+\left(48 p^{6}-144 p^{5}+168 p^{4}-96 p^{3}+36 p^{2}-12 p+1\right) \\
& t-4 p^{2}\left(4 p^{2}-4 p+3\right)(p-1)^{2},
\end{aligned}
$$




$$
\begin{aligned}
& g_{2}(1)=4\left(6 p^{2}-12 p+1\right) \\
& g_{3}(t)=3\left(16 p^{6}-48 p^{5}+72 p^{4}-64 p^{3}+36 p^{2}-12 p+1\right) t^{2}-24 p^{2}\left(4 p^{2}-4 p+3\right) \\
& (p-1)^{2} t+48 p^{6}-144 p^{5}+168 p^{4}-96 p^{3}+36 p^{2}-12 p+1, \\
& g_{3}(1)=4\left(6 p^{4}-12 p^{3}+18 p^{2}-12 p+1\right) \\
& \qquad g_{4}(t)=\left(16 p^{6}-48 p^{5}+72 p^{4}-64 p^{3}+36 p^{2}-12 p+1\right) t \\
& g_{4}(1)=12 p^{2}-24 p^{3}+24 p^{2}-12 p+1 .
\end{aligned}
$$

We divide the proof into two cases.

Case 1. $p=\lambda=\left(1+\sqrt{16 / \pi^{2}-1}\right) / 2$. Then equations (2.6), (2.11), (2.13), (2.15), and (2.17) lead to

$$
\begin{aligned}
& \lim _{t \rightarrow+\infty} f(t)=0 \\
& g_{1}(1)=-\frac{4\left(5 \pi^{2}-48\right)}{\pi^{2}}<0 \\
& g_{2}(1)=-\frac{2\left(5 \pi^{2}-48\right)}{\pi^{2}}<0 \\
& g_{3}(1)=-\frac{2\left(7 \pi^{4}-48 \pi^{2}-192\right)}{\pi^{4}}<0 \\
& g_{4}(1)=-\frac{2\left(\pi^{4}-96\right)}{\pi^{4}}<0 .
\end{aligned}
$$

Note that

$$
16 p^{6}-48 p^{5}+72 p^{4}-64 p^{3}+36 p^{2}-12 p+1=\frac{1024-\pi^{6}}{\pi^{6}}>0 .
$$

From (2.10), (2.12), (2.14), (2.16), and (2.23) we clearly see that

$$
\begin{aligned}
& \lim _{t \rightarrow+\infty} g_{1}(t)=+\infty \\
& \lim _{t \rightarrow+\infty} g_{2}(t)=+\infty \\
& \lim _{t \rightarrow+\infty} g_{3}(t)=+\infty \\
& \lim _{t \rightarrow+\infty} g_{4}(t)=+\infty
\end{aligned}
$$

From equation (2.16) and inequality (2.23) we clearly see that $g_{4}(t)$ is strictly increasing in $[1,+\infty)$, then inequality (2.22) and equation (2.27) lead to the conclusion that there exists $t_{0}>1$ such that $g_{4}(t)<0$ for $t \in\left(1, t_{0}\right)$ and $g_{4}(t)>0$ for $t \in\left(t_{0},+\infty\right)$. Hence, $g_{3}(t)$ is strictly decreasing in $\left[1, t_{0}\right]$ and strictly increasing in $\left[t_{0},+\infty\right)$. 
It follows from (2.21) and (2.26) together with the piecewise monotonicity of $g_{3}(t)$ that there exists $t_{1}>t_{0}>1$ such that $g_{2}(t)$ is strictly decreasing in $\left[1, t_{1}\right]$ and strictly increasing in $\left[t_{1},+\infty\right)$.

From (2.20) and (2.25) together with the piecewise monotonicity of $g_{2}(t)$ we conclude that there exists $t_{2}>t_{1}>1$ such that $g_{1}(t)$ is strictly decreasing in $\left[1, t_{2}\right]$ and strictly increasing in $\left[t_{2},+\infty\right)$.

Equations (2.7)-(2.9), (2.19), and (2.24) together with the piecewise monotonicity of $g_{1}(t)$ imply that there exists $t_{3}>t_{2}>1$ such that $f(t)$ is strictly decreasing in $\left[1, t_{3}\right]$ and strictly increasing in $\left[t_{3},+\infty\right)$.

Therefore, inequality (2.1) follows from equations (2.3)-(2.5) and (2.18) together with the piecewise monotonicity of $f(t)$.

Case 2. $p=\mu=(3+\sqrt{6}) / 6$. Then equation (2.10) becomes

$$
g_{1}(t)=\frac{\left(17 t^{2}+2 t+17\right)}{108}(t-1)^{2}>0
$$

for $t>1$.

Equations (2.7)-(2.10) and inequality (2.28) lead to the conclusion that $f(t)$ is strictly increasing in $[1,+\infty)$.

Therefore, inequality (2.2) follows from equations (2.3)-(2.5) and the monotonicity of $f(t)$.

From the monotonicity of $f(x)=S(x a+(1-x) b, x b+(1-x) a)$ in $[1 / 2,1]$ and inequalities (2.1) and (2.2) we know that inequality (1.3) holds for all $\alpha \leq\left(1+\sqrt{16 / \pi^{2}-1}\right) / 2, \beta \geq(3+\sqrt{6}) / 6$ and $a, b>0$ with $a \neq b$.

Next, we prove that $\lambda=\left(1+\sqrt{16 / \pi^{2}-1}\right) / 2$ is the best possible parameter in $(1 / 2,1)$ such that inequality (2.1) holds for all $a, b>0$ with $a \neq b$.

For any $1>p>\lambda=\left(1+\sqrt{16 / \pi^{2}-1}\right) / 2$, from (2.6) one has

$$
\lim _{t \rightarrow+\infty} f(t)=\frac{\pi}{2}-\frac{1}{p^{2}+(1-p)^{2}}>0 .
$$

Equations (2.3) and (2.4) together with inequality (2.29) imply that for any $1>p>\lambda=\left(1+\sqrt{16 / \pi^{2}-1}\right) / 2$ there exists $T_{0}=T_{0}(p)>1$ such that

$$
S(p a+(1-p) b, p b+(1-p) a)>T(a, b)
$$

for $a / b \in\left(T_{0},+\infty\right)$.

Finally, we prove that $\mu=(3+\sqrt{6}) / 6$ is the best possible parameter in $(1 / 2,1)$ such that inequality (2.2) holds for all $a, b>0$ with $a \neq b$.

For any $1 / 2<p<\mu=(3+\sqrt{6}) / 6$, from (2.11) one has

$$
g_{1}(1)=4\left(12 p^{2}-12 p+1\right)<0 .
$$

From inequality (2.30) and the continuity of $g_{1}(t)$ we know that there exists $\delta=\delta(p)$ $>0$ such that

$$
g_{1}(t)<0
$$

for $t \in(1,1+\delta)$. 
Equations (2.3)-(2.5) and (2.7)-(2.10) together with inequality (2.31) imply that for any $1 / 2<p<\mu=(3+\sqrt{6}) / 6$ there exists $\delta=\delta(p)>0$ such that

$$
T(a, b)>S(p a+(1-p) b, p b+(1-p) a)
$$

for $a / b \in(1,1+\delta)$.

\section{Acknowledgements}

This research was supported by the Natural Science Foundation of China under Grant 11071069, the Natural Science Foundation of Hunan Province under Grant 09JJ6003 and the Innovation Team Foundation of the Department of Education of Zhejiang Province under Grant T200924.

\section{Author details}

${ }^{1}$ Department of Mathematics and Computing Science, Hunan City University, Yiyang 413000, China ${ }^{2}$ Department of Mathematics, Hangzhou Normal University, Hangzhou 310012, China

\section{Authors' contributions}

Y-MC provided the main idea in this article. S-WH carried out the proof of inequality (2.1) in this article. Z-HS carried out the proof of inequality (2.2) in this article. All authors read and approved the final manuscript.

\section{Competing interests}

The authors declare that they have no competing interests.

Received: 19 September 2011 Accepted: 17 January 2012 Published: 17 January 2012

\section{References}

1. Seiffert, HJ: Aufgabe $\beta$ 16. Die Wurzel. 29, 221-222 (1995)

2. Neuman, E, Sándor, J: On the Schwab-Borchardt mean. Math Pannon. 14(2):253-266 (2003)

3. Neuman, E, Sándor, J: On the Schwab-Borchardt mean II. Math Pannon. 17(1):49-59 (2006)

4. Pahikkala, J: On contraharmonic mean and Phythagorean triples. Elem Math. 65(2):62-67 (2010)

5. Chu, Y-M, Wang, M-K, Qiu, Y-F: An optimal double inequality between power-type Heron and Seiffert means. J Inequal Appl 11 (2010). 2010, (Article ID 146945)

6. Wang, M-K, Qiu, Y-F, Chu, Y-M: Sharp bounds for Seiffert means in terms of Lehmer means. J Math Inequal. 4(4):581-586 (2010)

7. Chu, Y-M, Zong, C, Wang, G-D: Optimal convex combination bounds of Seiffert and geometric means for the arithmetic mean. J Math Inequal. 5(3):429-434 (2011)

8. Liu, H, Meng, X-J: The optimal convex combination bounds for the Seiffert's mean. J Inequal Appl 9 (2011). 2011, (Article ID 686384)

9. Hou, S-W, Chu, Y-M: Optimal convex combination bounds of root-square and harmonic root-square means for Seiffert mean. Int J Math Anal. 5(39):1897-1904 (2011)

10. Pillichshammer, F: On the root mean square weighted $L_{2}$ discrepancy of scrambled nets. J Complex. 20(5):638-653 (2004). doi:10.1016/j.jco.2003.08.007

11. Petitjean, M: On the root mean square quantitative chirality and quantitative symmetry measures. J Math Phys. 40(9):4587-4595 (1999). doi:10.1063/1.532988

12. Nelsen, RB: Proof without words: the harmonic mean-geometric mean-arithmetic mean-root mean square inequality. Math Mag. 60(3):158-158 (1987)

13. Abul-Magd, AY, Simbel, MH, Farag, MYH: Volume integrals and root-mean-square radii of the optical potential. Canad J Phys. 58(8):1230-1237 (1980). doi:10.1139/p80-162

14. Kamat, AR: On the mean successive difference and its ratio to the root mean square. Biometrika. 40, 116-127 (1953)

doi:10.1186/1029-242X-2012-11

Cite this article as: Chu et al: Sharp bounds for Seiffert mean in terms of root mean square. Journal of Inequalities and Applications 2012 2012:11. 